\title{
Simultaneous atomic force microscopy measurement of topography and contact resistance of metal films and carbon nanotubes
}

\author{
M. Stadermann, ${ }^{\text {a) }}$ H. Grube, ${ }^{\text {b) }}$ and J. J. Boland ${ }^{\text {() }}$ \\ Department of Chemistry, University of North Carolina at Chapel Hill, Chapel Hill, North Carolina 27599 \\ S. J. Papadakis \\ Department of Physics and Astronomy, University of North Carolina at Chapel Hill, Chapel Hill, \\ North Carolina 27599 \\ M. R. Falvo \\ Curriculum in Applied and Materials Science, University of North Carolina at Chapel Hill, Chapel Hill, \\ North Carolina 27599 \\ R. Superfine and S. Washburn \\ Department of Physics and Astronomy, Department of Computer Science, and Curriculum in Applied \\ and Materials Science, University of North Carolina at Chapel Hill, Chapel Hill, North Carolina 27599
}

(Received 14 April 2003; accepted 14 May 2003)

\begin{abstract}
We present a quartz tuning-fork-based atomic force microscopy (AFM) setup that is capable of mapping the surface contact resistance while scanning topography. The tuning-fork setup allows us to use etched Pt/Ir tips, which have higher durability and better conductivity than probes used in earlier AFM conductance measurements. The performance of the method is demonstrated with contact resistance measurements of gold lines on silicon dioxide and carbon nanotubes on graphite. (C) 2003 American Institute of Physics. [DOI: 10.1063/1.1590750]
\end{abstract}

\section{INTRODUCTION}

Since their first use ${ }^{1}$ as cantilevers in atomic force microscopy (AFM), ${ }^{2}$ quartz-crystal tuning forks have been used in a multitude of applications. Their high force sensitivity has enabled the resolution of subatomic features on silicon, ${ }^{3}$ and has allowed imaging in the attractive region of the tipsample potential even in air. ${ }^{4}$ These cantilevers also provide flexibility in the choice of probes, opening their use up to near-field scanning optical microscopy, ${ }^{5,6}$ and electrostatic force imaging. ${ }^{7}$

In this article, we expand the uses of tuning forks in scanning probe microscopy to conductance imaging AFM (CI AFM), which simultaneously measures local conductivity and topography. An etched Pt/Ir wire is used as the tip. We show that this method produces excellent topographical resolution as well as a reliable electrical contact. Earlier attempts at measuring conductance and topography simultaneously either employed metal coated tips ${ }^{8,9}$ or coatings of $\mathrm{NbN}$, doped diamond, or tungsten carbide. ${ }^{10,11}$ The solid metal tips used in this experiment are more durable and reliable than metal coated tips, which suffer from wear, and have a lower resistance than the other coatings. Most importantly, they also provide ohmic contact.

\section{DESIGN AND IMPLEMENTATION}

The force sensor in our experiment is a quartz-crystal tuning fork with a nominal resonance frequency $f_{\text {bare }}^{0}$

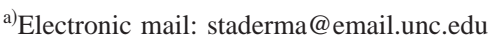

${ }^{\mathrm{b})}$ Present address: Materials Science and Technology, Los Alamos National Laboratory, MS G755, Los Alamos, NM 87545.

${ }^{c}$ Present address: Department of Chemistry, Trinity College, Dublin 2, Ireland.
}

$=32768 \mathrm{~Hz}$ and quality factor $Q_{\text {bare }} \sim 4 \times 10^{4} .{ }^{12}$ The fork is extracted from its hermetically sealed container and one of its prongs is fixed to a Macor mount in a manner similar to that of Giessibl ${ }^{13}$ using Stycast 2850FT epoxy. ${ }^{14}$ The epoxy was chosen for its high mechanical stiffness and short curing time. The free prong becomes a high- $Q$, self-exciting cantilever whose spring constant $k$ can be estimated from its dimensions (width $w=255 \mu \mathrm{m}$, thickness $t=100 \mu \mathrm{m}$, length $l=2.46 \mathrm{~mm})$ to be $k=0.25 E w(t / l)^{3} \approx 1330 \mathrm{~N} / \mathrm{m}$ where $E$ $=7.9 \times 10^{10} \mathrm{~N} / \mathrm{m}^{2}$ is Young's modulus of quartz. To this cantilever, an electrochemically etched $\mathrm{Pt} / \mathrm{Ir}$ tip ${ }^{15}$ (with a tip radius of $R_{\text {tip }} \sim 100 \mathrm{~nm}$ ) is attached using Stycast 2850FT, and then electrically connected to the leads on the prong using colloidal silver. ${ }^{16}$ The $Q$ and the resonance frequency drop during the process to $Q \sim 4000$ and $f_{\text {res }} \sim 13-15 \mathrm{kHz}$. This tip can be mounted in a conventional AFM. Our assembly mounts directly on the dither piezo of a Topometrix Discoverer AFM, but is easy to adapt to most AFMs.

Figure 1(a) is a schematic of the CI AFM. The selfexcitation loop and frequency detection are similar to those described by Giessibl. ${ }^{13}$ Cantilever motion is detected by measuring the potential difference between the two electrodes. A bias is applied to the sample. At the bottom of each oscillation of the tuning fork, the tip touches the surface, and current flows [Fig. 1(b)]. The current signal is converted to a voltage signal by a transimpedance amplifier. A gated integrator is used to sample only the peaks of the signal, giving us an estimate of the height of the peak and thus the maximum current through the contact. From the maximum current $I$, a contact conductance $G=I / V_{\text {bias }}$ can be extracted, where $V_{\text {bias }}$ is the voltage applied to the sample. Imaging is typically performed at a constant frequency shift $\Delta f$ (Ref. 

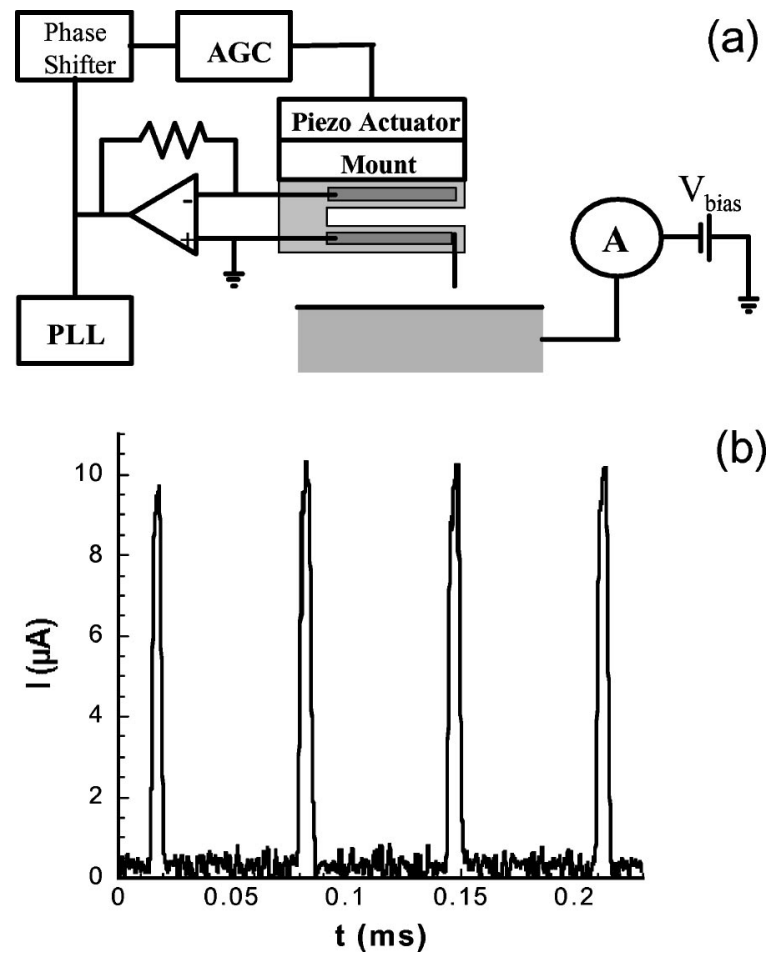

(b)

FIG. 1. (a) Experimental setup: The tuning fork is driven at resonance frequency using a self-excitation circuit consisting of an amplifier, a phase shifter, an automatic gain control, and a piezoactuator. The amplifier output is fed into a phase-locked loop frequency demodulator. Voltage is applied to the sample, and the current flow into the sample is measured. (b) Oscilloscope trace of the current as the tip scans over a gold line on silicon dioxide. The scan rate was $2 \mu \mathrm{m} / \mathrm{s}$

17) of $\Delta f=+2.3 \mathrm{~Hz}$ and an oscillation amplitude $A_{0}$ $=500-1000 \mathrm{~nm}$. The scan speed is 0.5 lines $/ \mathrm{s}$.

\section{RESULTS AND DISCUSSION}

Figure 1(b) shows an oscilloscope trace of the current as the tip passes over the sample. In the duration of the trace, the tip moves about $1 \mathrm{~nm}$ across the sample. The near constant peak height demonstrates the reproducibility of the
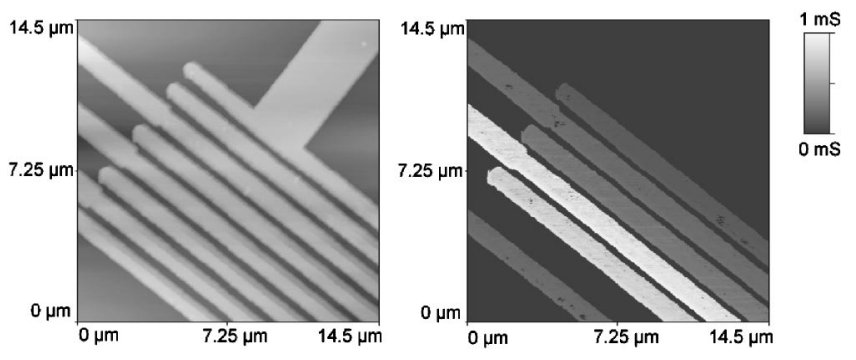

FIG. 2. Topographical (left-hand side) and conductance data (right-hand side) of $70 \mathrm{~nm}$ high gold leads on silicon dioxide taken simultaneously. The leads have varying contact resistances to the macroscopic leads and thus appear in different brightnesses. $50 \mathrm{mV}$ were applied to the sample. $A_{0}$ $=1000 \mathrm{~nm}, \Delta f=+2.3 \mathrm{~Hz}$.

measurement. The shape of the peaks indicates that the tip is in prolonged contact with the surface instead of barely making contact at the maximum tuning-fork displacement. The tip, however, does not seem to be crashing into the sample with enough force to cause obvious tip or sample deformation, since the scanning can be performed for hours without any degradation in quality of topographical or conductivity data.

A simultaneous measurement of current and topography is shown in Fig. 2. The sample consists of gold lines on a silicon dioxide surface. The gold lines are $70 \mathrm{~nm}$ high and fabricated using electron-beam lithography and are themselves connected to macroscopic gold leads made via photolithography. The contact resistance between the lines and the macroscopic leads varies, so that the absolute conductance measured at each of the lines is different. Some of the lines that appear on the topography image do not appear on the conductance image, because their resistance is too high. Increasing $V_{\text {bias }}$ makes these leads visible as well. Overall contact conductance to gold has been measured to be as high as $20 \mathrm{mS}$, but typically ranges between $1-10 \mathrm{mS}$.

To relate the microscopic measurement to macroscopic properties, the conductivity of gold is measured using the transmission line method. Each measurement along the gold
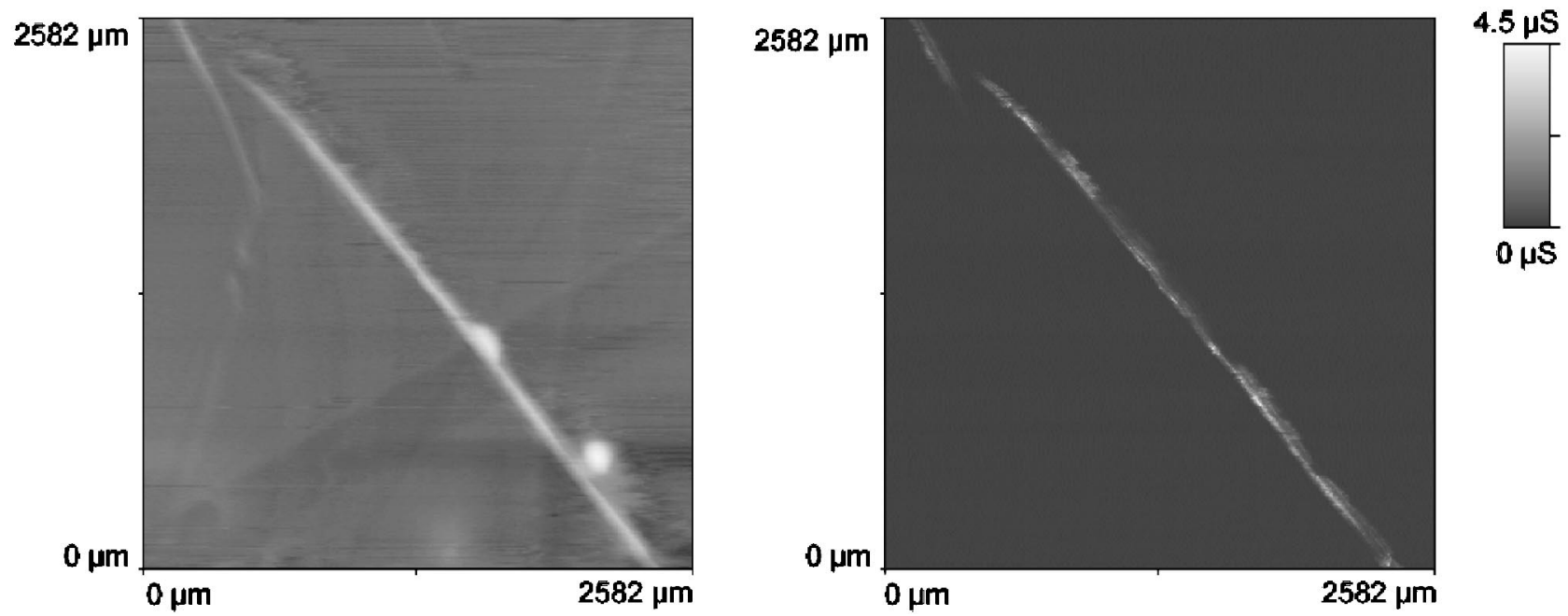

FIG. 3. Topographical (left-hand side) and conductance data (right-hand side) of multiwall nanotubes on HOPG. The MWNT have diameters of 2.5 nm and $5 \mathrm{~nm}$. A voltage of $50 \mathrm{mV}$ was applied to the sample. $A_{0}=800 \mathrm{~nm}, \Delta f=+2.3 \mathrm{~Hz}$. 
line is a data point and, from the slope of the fitted curve, we obtain the conductivity to be $110 \mathrm{kS} / \mathrm{cm}$. The literature value for conductivity in bulk is $446.1 \mathrm{kS} / \mathrm{cm}$. Since the line is an unannealed granular thin film, it is reasonable that the conductivity is lower.

The setup so far has been used to characterize conductances of multiwall nanotubes on highly oriented pyrolytic graphite (HOPG) (Fig. 3). Since the graphite only makes contact with the very end of the tip, its conductance appears lower than that of the nanotubes, which due to their geometry also touching the side of the tip and thus having a larger contact area. We were able to resolve changes in conductance on length scales of less than $100 \mathrm{~nm}$ under ambient conditions. Under more favorable high-vacuum conditions or with sharper tips, it should be possible to increase the resolution.

There are many possible applications of CI AFM. First, it provides a simple way of making two-point resistance measurements on systems that are not compatible with lithographic processes, such as $\mathrm{Li}_{2} \mathrm{Mo}_{6} \mathrm{Se}_{6}$ nanowires ${ }^{18}$ that oxidize in water or air, or biological samples that denature in contact with solvents. Three-terminal measurements ${ }^{19}$ can be performed with a mobile probe on samples that are contacted by two lithographically fabricated leads. Second, CI AFM allows reliable high-resolution imaging of resistance gradients and local electrical properties on thin metal films. Finally, as the contact force during the measurement is decreased, the contact between tip and sample changes from a macroscopic contact to a point contact. With a lithographically patterned contact as a second contact, the conducting probe can then be used as a mobile point contact for various techniques, such as transverse electron focusing. ${ }^{20}$ The injection angles of electrons coming from the tip is limited, making it possible to image the density of electron states in ballistically conducting samples. By further reducing the contact force and contact area, a quantum point contact should form once the dimensions of the contact approach the Fermi wavelength. $^{21,22}$

In conclusion, we have demonstrated the simultaneous imaging of topographical and local electrical properties using
AFM that we term CI AFM. The metal tip is a both durable and reliable contact. It solves the wear problem of earlier attempts at these measurements, and it provides a lowresistance ohmic contact to the sample. CI AFM shows great promise for two-point resistance measurements, local conductance imaging, and as mobile point contact probes.

\section{ACKNOWLEDGMENT}

This work was supported by the ONR-MURI program.

${ }^{1}$ H. Edwards, L. Taylor, W. Duncan, and A. J. Melmed, J. Appl. Phys. 82, 980 (1997).

${ }^{2}$ G. Binnig, C. Quate, and C. Gerber, Phys. Rev. Lett. 56, 930 (1986).

${ }^{3}$ F. Giessibl, S. Hembacher, H. Bielefeldt, and J. Mannhart, Science 289, 422 (2000).

${ }^{4}$ G. King, J. Lamb, and G. Nunes, Appl. Phys. Lett. 79, 1712 (2001).

${ }^{5}$ P. Güethner, U. Fisher, and K. Dransfeld, Appl. Phys. B: Lasers Opt. 48, 89 (1989).

${ }^{6}$ K. Karrai and R. Grober, Appl. Phys. Lett. 66, 1842 (1995).

${ }^{7}$ Z. Wang, J. Bao, H. Zhang, and W. Guo, Appl. Phys. Lett. 81, 1300 (2002).

${ }^{8}$ F. Houzé, R. Meyer, O. Schneegans, and L. Boyer, Appl. Phys. Lett. 69, 1975 (2000).

${ }^{9}$ M. Lantz, S. O'Shea, and M. Welland, Phys. Rev. B 56, 15345 (1997).

${ }^{10}$ H. Dai, E. Wong, and C. Lieber, Science 272, 523 (1996).

${ }^{11}$ M. Freitag, M. Radosavljević, W. Clauss, and A. Johnson, Phys. Rev. B 62, R2307 (2000).

${ }^{12}$ Digi-Key 300-1003-ND.

${ }^{13}$ F. Giessibl, Appl. Phys. Lett. 73, 3956 (1998).

${ }^{14}$ Emerson \& Cuming Stycast 2850FT, Catalyst 24LV.

${ }^{15} 0.005$ in. $\mathrm{Pt} / \mathrm{Ir} 95: 5$ wire, ac etched at $1.2 \mathrm{~V}, 1 \mathrm{kHz}$ in $3 \mathrm{~m} \mathrm{KCN} / 1 \mathrm{M} \mathrm{NaOH}$ solution.

${ }^{16}$ Prod. No. 16031, Ted Pella, Inc., P.O. Box 492477, Redding, CA 960492477.

${ }^{17}$ T. Albrecht, P. Grütter, D. Horne, and D. Rugar, J. Appl. Phys. 69, 668 (1991).

${ }^{18}$ M. Potel, R. Chevrel, M. Sergent, J. Armici, M. Decroux, and O. Fischer, J. Solid State Chem. 35, 286 (1980).

${ }^{19}$ M. Büttiker, Phys. Rev. B 35, 4123 (1986).

${ }^{20}$ V. Tsoi, J. Bass, and P. Wyder, Rev. Mod. Phys. 71, 1641 (1999).

${ }^{21}$ B. van Wees, H. van Houten, C. Beenakker, J. Williamson, L. Kouwenhoven, D. van der Marel, and C. Foxon, Phys. Rev. Lett. 60, 848 (1988).

${ }^{22}$ D. Wharam, T. Thornton, R. Newbury, M. Pepper, H. Ahmed, J. Frost, D. Hasko, D. Peacock, D. Ritchie, and G. Jones, J. Phys. C 21, L887 (1988). 